\title{
EFFECT OF N LEVELS AND SOME PLANT GROWTH REGULATORS ON HYBRID RICE (ORYZA SATIVA L.) SEED PRODUCTION
}

\author{
HAMAD, H. SH. ${ }^{1}$ - GHONEIM, A. M..$^{2 *}$ ZIDAN, A. A. ${ }^{1}-$ GOMAA, M. A. ${ }^{1}$ \\ ${ }^{1}$ Rice Research and Training Center, Kafr El-Sheikh, Field Crops Research Institute, \\ Agricultural Research Center, Egypt \\ ${ }^{2}$ Field Crops Research Institute, Agricultural Research Center, Giza 12112, Egypt \\ *Corresponding author \\ e-mail: adelrrtc.ghoneim@gmail.com; phone: +20-10-9963-7222
}

(Received $15^{\text {th }}$ Mar 2021; accepted $10^{\text {th }}$ Jun 2021)

\begin{abstract}
The aim of the present investigation is to identify the suitable $\mathrm{N}$ application rates and gibberellic acid to enhance hybrid rice seed production in Egypt. The experimental material comprising of both the parent Giza 178R and CMS line G46A was treated with different N levels and foliar Gibberellic acid $\left(\mathrm{GA}_{3}\right)$. Treatments were arranged in a split-plot design with three replications. The main plots were devoted to the four $\mathrm{N}$ fertilizer levels $\left(0,55,110\right.$ and $\left.165 \mathrm{~kg} \mathrm{~N} \mathrm{ha}^{-1}\right)$, while, the doses of foliar $\mathrm{GA}_{3}+$ boric acid were $0,150,150+0.5 \%, 200,200+0.5 \%, 300$ and $300+0.5 \% \mathrm{~g} \mathrm{ha}^{-1}$ arranged in the sub plots. Application of $165 \mathrm{~kg} \mathrm{~N}^{\mathrm{g}} \mathrm{a}^{-1}$ produced the highest seed yield in both seasons. The application rates of $300 \mathrm{~g} \mathrm{GA}_{3}+0.5 \%$ boric acid ha ${ }^{-1}$ produced the highest seed yield. The interaction between $\mathrm{N}$ levels and doses of $\mathrm{GA}_{3}$ was highly significant for duration of spikelet opening, spikelet opening angle, stigma exsertion, plant height, panicle exsertion, panicle length, number of fertile tillers, panicle weight, seed set, harvest index and seed yield. It is concluded that, the treatment combination of $\left(165 \mathrm{~kg} \mathrm{~N} \mathrm{ha}^{-1}\right.$ and $300 \mathrm{~g} \mathrm{GA}_{3} \mathrm{ha}^{-1}+0.5 \%$ boric acid) gave the best effects for most of the rice characteristics.
\end{abstract}

Keywords: hybrid rice, high yield breeding, CMS, nitrogen fertilizer, gibberellic acid, seed yield

\section{Introduction}

Rice is the most important food crop in Egypt, contributing about $21 \%$ of the total cereal consumption and utilization about $18 \%$ of the annual water consumption (RRTC, 2018). In Egypt, the average national rice yield has to be increased by 25$30 \%$ to meet the demands of the rising population. This seems difficult considering to narrow gap between potential and actual yield. However, among available technologies the increase yield, is the exploitation of heterosis of hybrid rice, which appears to be practical approach for Egypt (Bastawisi et al., 2002).

Hybrid rice technology is the innovative breakthrough that can further increase rice production leading to food security and reduction of poverty in Egypt. Hybrid rice varieties can out yield conventional cultivars by at least $15 \%$ under the same input levels. Hence, this technology can be used to break the current yield plateau in rice, where yield levels of the conventional released cultivars have stabilized. Hybrid rice seed production technology has increased rice yield potential by $10-20 \%$ in Egypt over the past 20 years (Hamad, 2018). Hybrid seed rice production must be produced based on hybrid male sterile (CMS), since, rice is a self-pollinated. Most of the CMS parents developed in Egypt are based on CMS lines having the wild abortive (WA) backgrounds. Incomplete panicle exsertion in almost all the (WA) based CMS lines is one of the major impediments in obtaining higher seed yields as about 20-30\% of the panicles are enclosed in the flag leaf, and the enclosed spikelets are not available for 
cross pollination, thus resulting in lower seed yields. To increases the hybrid seed rice production by improving the out-crossing CMS lines (Shi-Hua et al., 2006; Zheng et al., 2018).

Plant hormones play a vital role in coordination of many growth and behavioral processes in the plant life. They regulate the amount, type and direction of plant growth. Remarkable application of Plant Growth Regulators (PGR) such as manipulating plant developments, enhancing yield and quality have been actualized in recent years (Yuan, 2007, 2010). Plant hormones including auxins, gibberellins and cytokinin are involved in controlling developmental of cell division, cell elongation and protein synthesis. Plants have the ability to store excessive amounts of exogenously supplied hormones in the form of reversible conjugates which release active hormone when and where plant needs them during the growth period. Auxins (i.e., NAA) and gibberellins (i.e., $\mathrm{GA}_{3}$ ) being well known plant growth promoting hormones has shown to be involved in a variety of plant growth and development processes (Li et al., 2009; Xie et al., 2011). Gibberellic acid (GA3), plays diverse roles in the stimulation and synchronization of flowering, seed development, seed weight (Zhu et al., 2015; Garcia et al., 2018). In addition, Parihar et al. (2012) found that GA 3 application resulted in a better seed set for seed production of a CMS line of hybrid rice.

Nitrogen $(\mathrm{N})$ fertilizer is more urgent for security hybrid rice production. Applying sufficient $\mathrm{N}$ level is essential not only for getting greatest economic return, but also to reducing the environmental impact (Fageria and Baligar, 2003; Gewaily et al., 2018). Modern high-yielding rice cultivars may have differences in accumulation and utilize $\mathrm{N}$ from the soil and fertilizer application. The recovery of applied $\mathrm{N}$ fertilizer for rice is low and deterrent to get the full potential yield. So, it is necessary to identify what the optimal dose needed for each cultivar as well as its influence on yield and its attributes. Shaiful et al. (2009) reported that, using proper N level led to spare money and maintain our environment sound. Moreover, the abundant application of $\mathrm{N}$ fertilizer has an influence on the soil and the environment through the fertilizer residual effect. Apply the most suitable dose of $\mathrm{N}$ fertilization is a main interest of economic viability of hybrid rice production (Shaiful et al., 2009; Ghoneim, 2020).

Therefore, the aim of the present instigation is to identify a suitable $\mathrm{N}$ levels and $\mathrm{GA}_{3}$ on some important yield characteristics of the CMS line G46A crossed by the restorer line Giza $178 \mathrm{R}$ which used in producing the hybrid seed in Egypt.

\section{Materials and methods}

\section{Field experiment}

Two field experiments were conducted at Sakha agricultural experimental farm, Kafr El-Sheikh Governorate, Egypt (31 $09^{\circ} \mathrm{N}$ Latitude and $3068^{\circ}$ longitude) during 2019 and 2020 rice growing seasons. Treatments were arranged in a split-plot design with three replications. The main plots were devoted to the four $\mathrm{N}$ fertilizer levels $(0$, 55,110 and $165 \mathrm{~kg} \mathrm{~N} \mathrm{ha}^{-1}$ ), while, the doses of $\mathrm{GA}_{3}+$ boric acid combinations were $\left(0,150,150+0.5 \%, 200,200+0.5 \%, 300\right.$ and $300+0.5 \% \mathrm{~g} \mathrm{ha}^{-1}$ were arranged in the sub plots with plot size of $12.5 \mathrm{~m}^{2}$. Rice seeds at the rate of $20 \mathrm{~kg} \mathrm{ha}^{-1}$ (15 kg of CMS and $5 \mathrm{~kg}$ of restorer line) were soaked in fresh water for $24 \mathrm{~h}$ and then, incubated for $48 \mathrm{~h}$ to hasten germination. The male parent Giza $178 \mathrm{R}$ was sown on May $1^{\text {st }}$ which is sixteen days earlier than the CMS line G46A (as female parent) to get a proper 
synchronization of flowering. Nitrogen fertilizer rates were applied as Urea $(46.5 \% \mathrm{~N})$ in three split applications. The first dose was applied as basal application, while the second dose at 25 days after transplanting (DAT) and the third dose application at 45 DAT. Seedlings were carefully pulled from the nursery after 30 days from seeding and transferred to the permanent field. Seedlings were hand transplanted in hills at the rate of 2-3 seedlings hill ${ }^{-1}$. The row spacing maintained for R-R, R-A and A-A lines were 20, 30, and $15 \mathrm{~cm}$, respectively. Hill spacing for both $\mathrm{R}$ and $\mathrm{A}$ lines were maintained at $15 \mathrm{~cm}$. Isolation space of $100 \mathrm{~m}$ was considered for CMS seed production. Moreover, the experimental field was surrounded by an additional 20 rows of $\mathrm{R}$ lines to avoid any possibility of cross pollination. The sub plots were isolated by plastic barrier (2.5 m height) to avoid any pollen grain movement from treatment to another.

\section{Foliar application of growth regulators}

Application of $\mathrm{GA}_{3}$ and boric acid was done in two splits. The first split consisted of $40 \%$ of the total amount applied when A and R line was at 15-20\% heading and the second spray (60\%) was applied when A and $\mathrm{R}$ line was at $35-40 \%$ heading. Rice is basically a self-pollinated crop. Supplementary pollination serves to enhance the outcrossing rate in order to increase seed set. Supplementary pollination was done by shaking the pollen parents ( $\mathrm{R}$ line) with bamboo sticks. This operation was done 2-3 times in between 9 am to 11.30 am and be continued for 10-12 days during flowering.

\section{Measurements of seed yield and its components}

At maturity, 10 hills were sampled diagonally from a $5 \mathrm{~m}^{2}$ harvest area to determine the yield components. Days to heading, duration of spikelet opening (min), spikelet opening angle $\left({ }^{\circ}\right)$, stigma exsertion $(\%)$, plant height $(\mathrm{cm})$, panicle exsertion $(\%)$, panicle length $(\mathrm{cm})$, number of fertile tillers hill ${ }^{-1}$, panicle weight $(\mathrm{g})$, seed set $(\%)$, harvest index $(\%)$ and seed yield $\left(\mathrm{t} \mathrm{ha}^{-1}\right)$ were recorded. The hybrid rice seed production yield was determined from a $5 \mathrm{~m}^{2}$ area in each plot and adjusted to $14 \%$ moisture content.

Panicle exsertion (\%) was estimated from the ten panicles and calculated as follows:

$$
\text { Panicle exsertion }(\%)=\frac{\text { Exerted panicle length }}{\text { Panicle length }} \times 100
$$

Fertility (\%) was estimated as an average from the ten panicles and calculated as follows:

\section{Statistical analysis}

$$
\begin{aligned}
& \text { Fertility } \%=\frac{\text { Number of filled grains per panicle }}{\text { Total spikelet's perpanicle }} \times 100 \\
& \text { Harvest index }(\text { H.I } \%)=\frac{\text { Grain yield }}{\text { Biological yield }} \times 100
\end{aligned}
$$

The statistical analysis was done using analysis of variance technique by means of Genes computer software package (Gomez and Gomez, 1984). Means of treatments were compared using the Duncan's multiple range test with a 5\% probability level. 


\section{Results}

\section{Nitrogen levels effect}

The effect of $\mathrm{N}$ levels, doses of $\mathrm{GA}_{3}$ application and their interaction on days to heading, duration of spikelet opening (min), spikelet opening angle $\left(^{\circ}\right)$ and stigma exsertion (\%) are presented in Table 1. The highest values for days to heading, duration of spikelet opening, spikelet opening angle and stigma exsertion were obtained with the application rate of $165 \mathrm{~kg} \mathrm{~N} \mathrm{ha}^{-1}$ in both seasons. While, the lowest values for days to heading, duration of spikelet opening, spikelet opening angle and stigma exsertion were recorded under control treatment in both seasons.

Table 1. Effect of $N$ levels and $G A_{3}+B$.A. foliar application and their interaction on floral traits during 2019 and 2020 season

\begin{tabular}{|c|c|c|c|c|c|c|c|c|}
\hline \multirow{2}{*}{$\begin{array}{l}\text { Main effect and } \\
\text { interaction }\end{array}$} & \multicolumn{2}{|c|}{$\begin{array}{l}\text { Days to } 50 \% \\
\text { heading }\end{array}$} & \multicolumn{2}{|c|}{$\begin{array}{c}\text { Duration of spikelet } \\
\text { opening (min) }\end{array}$} & \multicolumn{2}{|c|}{$\begin{array}{c}\text { Spikelet opening } \\
\text { angle }\left(^{\circ}\right)\end{array}$} & \multicolumn{2}{|c|}{$\begin{array}{c}\text { Stigma exsertion } \\
(\%)\end{array}$} \\
\hline & 2019 & 2020 & 2019 & 2020 & 2019 & 2020 & 2019 & 2020 \\
\hline \multicolumn{9}{|l|}{$\mathrm{N}$ levels $\left(\mathrm{kg} \mathrm{N} \mathrm{ha} \mathbf{~}^{-1}\right)$} \\
\hline 0 & $82.90 \mathrm{~d}$ & $83.01 d$ & $135.19 \mathrm{~d}$ & $137.43 d$ & $23.26 \mathrm{~d}$ & $24.77 \mathrm{~d}$ & $54.89 \mathrm{~d}$ & $56.14 d$ \\
\hline 55 & $84.63 c$ & $85.04 \mathrm{c}$ & $144.42 \mathrm{c}$ & $147.09 \mathrm{c}$ & $25.68 \mathrm{c}$ & $26.71 \mathrm{c}$ & $57.48 \mathrm{c}$ & $59.38 \mathrm{c}$ \\
\hline 110 & $85.65 b$ & $86.48 b$ & $148.47 b$ & $150.38 b$ & $27.76 b$ & $28.49 \mathrm{~b}$ & $61.77 b$ & $63.75 b$ \\
\hline 165 & $86.90 \mathrm{a}$ & $87.98 \mathrm{a}$ & $152.67 \mathrm{a}$ & $155.47 \mathrm{a}$ & $29.63 a$ & $30.67 \mathrm{a}$ & $65.44 \mathrm{a}$ & $67.70 \mathrm{a}$ \\
\hline F-test & $* *$ & $* *$ & $* *$ & $* *$ & $* *$ & $* *$ & $* *$ & $* *$ \\
\hline \multicolumn{9}{|c|}{ GA3 + B.A $\left(\mathrm{g} \mathrm{ha}^{-1}\right)$ dosage (D) } \\
\hline 0 & 84.89 & 85.78 & $116.00 f$ & $119.83 \mathrm{~g}$ & $20.91 \mathrm{~g}$ & $21.87 \mathrm{~g}$ & $50.85 \mathrm{~g}$ & $52.18 \mathrm{~g}$ \\
\hline 150 & 84.86 & 85.63 & $130.66 \mathrm{e}$ & $133.00 \mathrm{f}$ & $24.32 \mathrm{f}$ & $24.92 f$ & $55.23 \mathrm{f}$ & $56.52 f$ \\
\hline $150+0.5 \%$ & 84.82 & 85.65 & $149.50 \mathrm{~d}$ & $152.25 \mathrm{~d}$ & $26.49 d$ & $27.80 \mathrm{~d}$ & $58.93 d$ & $60.54 d$ \\
\hline 200 & 84.81 & 85.52 & $149.33 d$ & $150.58 \mathrm{e}$ & $26.12 \mathrm{e}$ & $27.08 \mathrm{e}$ & $58.40 \mathrm{e}$ & $59.95 \mathrm{e}$ \\
\hline $200+0.5 \%$ & 85.08 & 85.53 & $156.25 b$ & $158.08 b$ & $28.95 b$ & $30.05 b$ & $63.49 b$ & $66.02 b$ \\
\hline 300 & 84.94 & 85.7 & $154.33 c$ & $155.91 \mathrm{c}$ & $28.52 \mathrm{c}$ & $29.34 \mathrm{c}$ & $63.00 \mathrm{c}$ & $65.47 \mathrm{c}$ \\
\hline $300+0.5 \%$ & 85.07 & 85.06 & $160.26 a$ & $163.50 \mathrm{a}$ & $31.41 \mathrm{a}$ & $32.54 \mathrm{a}$ & $69.37 \mathrm{a}$ & $71.52 \mathrm{a}$ \\
\hline F-test & NS & NS & $* *$ & $* *$ & $* *$ & $* *$ & $* *$ & $* *$ \\
\hline \multicolumn{9}{|l|}{ Interaction } \\
\hline$(\mathrm{N} \times \mathrm{D})$ & NS & NS & $* *$ & $* *$ & $* *$ & $* *$ & $* *$ & $* *$ \\
\hline
\end{tabular}

$*$ = Significant at 0.05 level, $* *=$ Significant at 0.01 level and NS = Not significant. Means in the same column designated by the same letter are not significantly different at $5 \%$ level

\section{Growth regulators effect}

Otherwise, there were highly significant differences on the effect of $\mathrm{GA}_{3}$ and boric acid application on duration of spikelet opening, spikelet opening angle and stigma exsertion in both seasons (Table 1). The highest values for duration of spikelet opening, spikelet opening angle and stigma exsertion were obtained with the $300 \mathrm{~g}$ $\mathrm{GA}_{3}+0.5 \%$ B.A ha ${ }^{-1}$ in both seasons, while, the lowest values for duration of spikelet opening, spikelet opening angle and stigma exsertion were obtained under control treatment in both seasons. 


\section{Interaction effect}

The interaction between nitrogen levels and doses of $\mathrm{GA}_{3}$ application was highly significant for duration of spikelet opening, spikelet opening angle and stigma exsertion in both seasons. While, it is not significantly for days to $50 \%$ heading in both seasons. The results in Table 2 showed that the treatment of $165 \mathrm{~kg} \mathrm{~N}^{-1}$ with $300 \mathrm{~g} \mathrm{GA}_{3}+0.5 \%$ B.A ha ${ }^{-1}$ combination, recorded significantly highest values of duration of spikelet opening, spikelet opening angle and stigma exsertion in both seasons.

Table 2. Effect of N levels and $G A_{3}+B . A$. dosage, as well as their interaction on floral traits during 2019 and 2020 seasons

\begin{tabular}{|c|c|c|c|c|c|c|c|}
\hline \multirow{2}{*}{$\begin{array}{c}\text { N levels } \\
\left(\text { kg N ha-1) }^{-1}\right.\end{array}$} & \multirow{2}{*}{$\begin{array}{l}\mathbf{G A} A_{3}+\mathbf{B} \cdot \mathbf{A} \\
\left(\mathrm{g} \mathrm{ha}^{-1}\right)(\mathbf{D})\end{array}$} & \multicolumn{2}{|c|}{$\begin{array}{l}\text { Duration of spikelet } \\
\text { opening (min) }\end{array}$} & \multicolumn{2}{|c|}{ Spikelet opening angle $\left(^{\circ}\right)$} & \multicolumn{2}{|c|}{ Stigma exsertion (\%) } \\
\hline & & 2019 & 2020 & 2019 & 2020 & 2019 & 2020 \\
\hline \multirow{7}{*}{0} & 0 & $110.00 \mathrm{p}$ & $115.33 \mathrm{t}$ & 19.391 & $20.27 \mathrm{~s}$ & $49.66 \mathrm{q}$ & $50.49 \mathrm{r}$ \\
\hline & 150 & $118.67 n$ & $121.66 \mathrm{q}$ & $21.52 \mathrm{j}$ & $22.09 \mathrm{q}$ & $52.30 n$ & 53.760 \\
\hline & $150+0.5 \%$ & $135.33 \mathrm{k}$ & $138.33 \mathrm{~m}$ & 23.05hi & $24.77 \mathrm{~m}$ & $54.62 \mathrm{~m}$ & $55.72 \mathrm{n}$ \\
\hline & 200 & $139.67 \mathrm{j}$ & $137.33 n$ & 23.01hi & $24.08 n$ & $54.21 \mathrm{~m}$ & $55.29 \mathrm{n}$ \\
\hline & $200+0.5 \%$ & $147.48 \mathrm{~h}$ & $148.33 \mathrm{j}$ & $25.53 \mathrm{~g}$ & $26.84 \mathrm{k}$ & $56.73 \mathrm{k}$ & $57.92 \mathrm{k}$ \\
\hline & 300 & $144.26 \mathrm{i}$ & $146.66 \mathrm{k}$ & $25.09 \mathrm{~g}$ & 26.121 & $56.17 \mathrm{k}$ & $57.161 \mathrm{~m}$ \\
\hline & $300+0.5 \%$ & $150.30 \mathrm{~g}$ & $154.33 \mathrm{~h}$ & $27.84 \mathrm{e}$ & $29.20 \mathrm{~g}$ & $60.56 h$ & $62.65 \mathrm{~g}$ \\
\hline \multirow{7}{*}{55} & 0 & 115.350 & $118.33 \mathrm{~s}$ & $20.10 \mathrm{k}$ & $21.50 \mathrm{r}$ & $50.20 p$ & $51.61 \mathrm{q}$ \\
\hline & 150 & 131.001 & 133.330 & $23.56 \mathrm{~h}$ & $24.22 n$ & $54.35 \mathrm{~m}$ & $55.53 n$ \\
\hline & $150+0.5 \%$ & $150.26 \mathrm{~g}$ & $154.66 \mathrm{~h}$ & $25.72 \mathrm{~g}$ & $26.81 \mathrm{k}$ & $56.72 \mathrm{k}$ & $57.68 \mathrm{kl}$ \\
\hline & 200 & $148.33 h$ & $152.33 \mathrm{i}$ & $25.21 \mathrm{~g}$ & 26.151 & $56.15 \mathrm{k}$ & $57.26 \mathrm{klm}$ \\
\hline & $200+0.5 \%$ & $154.31 \mathrm{f}$ & $156.33 \mathrm{~g}$ & $27.58 \mathrm{e}$ & $28.75 \mathrm{~h}$ & $58.68 \mathrm{ij}$ & $61.87 \mathrm{~h}$ \\
\hline & 300 & $153.33 \mathrm{f}$ & $154.33 \mathrm{~h}$ & $27.12 \mathrm{ef}$ & $28.18 \mathrm{i}$ & $58.52 \mathrm{ij}$ & $61.06 \mathrm{i}$ \\
\hline & $300+0.5 \%$ & $158.30 \mathrm{~d}$ & $160.33 \mathrm{e}$ & $30.46 c$ & $31.31 d$ & $67.74 \mathrm{e}$ & $70.65 d$ \\
\hline \multirow{7}{*}{110} & 0 & $118.33 n$ & $120.33 r$ & $21.53 \mathrm{i}$ & $22.38 \mathrm{p}$ & $51.37 \mathrm{o}$ & $52.84 \mathrm{p}$ \\
\hline & 150 & $134.66 \mathrm{k}$ & $136.66 n$ & $25.56 \mathrm{~g}$ & 26.181 & 55.411 & $56.90 \mathrm{~m}$ \\
\hline & $150+0.5 \%$ & $154.60 f$ & $156.33 \mathrm{~g}$ & $27.47 \mathrm{e}$ & $28.83 \mathrm{~h}$ & $58.86 \mathrm{i}$ & $60.72 \mathrm{i}$ \\
\hline & 200 & $153.00 \mathrm{f}$ & $154.33 \mathrm{~h}$ & $27.10 \mathrm{ef}$ & $28.09 \mathrm{i}$ & $58.18 \mathrm{j}$ & $60.12 j$ \\
\hline & $200+0.5 \%$ & $158.30 \mathrm{~d}$ & $160.33 \mathrm{e}$ & $30.21 \mathrm{c}$ & $30.78 \mathrm{e}$ & $67.90 \mathrm{e}$ & $70.54 d$ \\
\hline & 300 & $156.62 \mathrm{de}$ & $158.33 \mathrm{f}$ & $29.84 \mathrm{~cd}$ & $30.02 \mathrm{f}$ & $67.22 \mathrm{f}$ & $70.29 d$ \\
\hline & $300+0.5 \%$ & 163.67be & $166.33 c$ & $32.64 b$ & $33.18 \mathrm{c}$ & $73.50 \mathrm{~b}$ & $74.83 b$ \\
\hline \multirow{7}{*}{165} & 0 & $120.33 \mathrm{~m}$ & $125.33 p$ & $22.60 \mathrm{i}$ & 23.350 & $52.16 n$ & 53.780 \\
\hline & 150 & $138.33 \mathrm{j}$ & 140.331 & $26.61 f$ & $27.16 \mathrm{j}$ & $58.86 \mathrm{i}$ & $59.86 \mathrm{j}$ \\
\hline & $150+0.5 \%$ & $157.66 \mathrm{de}$ & $159.66 \mathrm{e}$ & $29.75 \mathrm{~cd}$ & $30.80 \mathrm{e}$ & $65.52 \mathrm{~g}$ & $68.03 \mathrm{e}$ \\
\hline & 200 & $156.33 \mathrm{e}$ & $158.33 \mathrm{f}$ & $29.11 d$ & $30.02 \mathrm{f}$ & $65.08 \mathrm{~g}$ & $67.14 f$ \\
\hline & $200+0.5 \%$ & $164.66 b$ & $167.33 b$ & $32.47 b$ & $33.81 \mathrm{~b}$ & $70.64 c$ & $73.75 c$ \\
\hline & 300 & $162.66 \mathrm{c}$ & $164.33 d$ & $32.03 \mathrm{~b}$ & $33.03 \mathrm{c}$ & $70.11 d$ & $73.37 \mathrm{c}$ \\
\hline & $300+0.5 \%$ & $168.66 \mathrm{a}$ & $173.00 \mathrm{a}$ & $34.78 \mathrm{a}$ & $36.47 \mathrm{a}$ & $75.68 \mathrm{a}$ & $77.94 \mathrm{a}$ \\
\hline
\end{tabular}

$*$ = Significant at 0.05 level, $* *=$ Significant at 0.01 level and NS = Not significant. Means in the same column designated by the same letter are not significantly different at $5 \%$ level 


\section{Nitrogen levels effect}

The effect of $\mathrm{N}$ levels, doses of $\mathrm{GA}_{3}$ application and their interaction on plant height $(\mathrm{cm})$, panicle exsertion $(\%)$, panicle length $(\mathrm{cm})$ and number of fertile tillers hill $^{-1}$ are given in Table 3. The highest values for plant height, panicle exsertion, panicle length and number of fertile tillers hill $^{-1}$ were obtained with application of 165 $\mathrm{kg} \mathrm{N} \mathrm{ha}{ }^{-1}$ in both seasons while, the lowest one was found under control treatment in both seasons.

Table 3. Mean performance of various quantitative traits of rice under different $N$ levels and GA3 during 2019 and 2020 seasons

\begin{tabular}{|c|c|c|c|c|c|c|c|c|}
\hline \multirow{2}{*}{$\begin{array}{l}\text { Main effect and } \\
\text { interaction }\end{array}$} & \multicolumn{2}{|c|}{$\begin{array}{c}\text { Plant height } \\
(\mathrm{cm})\end{array}$} & \multicolumn{2}{|c|}{$\begin{array}{c}\text { Panicle exertion } \\
(\%)\end{array}$} & \multicolumn{2}{|c|}{$\begin{array}{c}\text { Panicle length } \\
(\mathrm{cm})\end{array}$} & \multicolumn{2}{|c|}{$\begin{array}{c}\text { Number of fertile } \\
\text { tillers } \text { hill }^{-1}\end{array}$} \\
\hline & 2019 & 2020 & 2019 & 2020 & 2019 & 2020 & 2019 & 2020 \\
\hline \multicolumn{9}{|l|}{$\mathrm{N}$ levels $\left(\mathrm{kg} \mathrm{N} \mathrm{ha}^{-1}\right)$} \\
\hline 0 & $83.51 \mathrm{~d}$ & $85.47 \mathrm{~d}$ & $60.00 \mathrm{~d}$ & $59.68 d$ & $16.68 \mathrm{~d}$ & $17.69 \mathrm{~d}$ & $12.65 d$ & $14.01 d$ \\
\hline 55 & $85.29 \mathrm{c}$ & $86.49 \mathrm{c}$ & $60.89 \mathrm{c}$ & $61.85 c$ & $17.90 \mathrm{c}$ & $18.48 \mathrm{c}$ & $14.74 \mathrm{c}$ & $15.12 \mathrm{c}$ \\
\hline 110 & $88.80 \mathrm{~b}$ & $90.53 b$ & $62.68 b$ & $63.03 b$ & $19.02 b$ & $19.70 \mathrm{~b}$ & $16.88 \mathrm{~b}$ & $17.65 b$ \\
\hline 165 & $95.76 \mathrm{a}$ & $96.34 \mathrm{a}$ & $64.18 \mathrm{a}$ & $64.44 \mathrm{a}$ & $20.31 \mathrm{a}$ & $20.73 a$ & $18.85 \mathrm{a}$ & $19.22 \mathrm{a}$ \\
\hline F-test & $* *$ & $* *$ & $* *$ & $* *$ & $* *$ & $* *$ & $* *$ & $* *$ \\
\hline \multicolumn{9}{|c|}{$\mathrm{GA}_{3}+$ B.A $\left(\mathrm{g} \mathrm{ha}^{-1}\right)$ dosage (D) } \\
\hline 0 & $80.13 \mathrm{~g}$ & $81.71 \mathrm{~g}$ & $34.10 \mathrm{~g}$ & $34.81 \mathrm{~g}$ & $16.59 \mathrm{~g}$ & $17.20 \mathrm{~g}$ & $12.25 \mathrm{~g}$ & $12.84 \mathrm{~g}$ \\
\hline 150 & 84.99f & $85.35 \mathrm{f}$ & $57.89 f$ & $58.78 \mathrm{f}$ & $17.47 \mathrm{f}$ & $17.99 f$ & $13.70 \mathrm{f}$ & $14.25 f$ \\
\hline $150+0.5 \%$ & $88.08 \mathrm{~d}$ & $88.93 d$ & $66.39 \mathrm{~d}$ & $65.35 d$ & $17.93 \mathrm{~d}$ & $18.86 \mathrm{~d}$ & $15.51 d$ & $16.00 \mathrm{~d}$ \\
\hline 200 & $87.47 \mathrm{e}$ & $88.37 \mathrm{f}$ & $65.05 \mathrm{e}$ & $64.29 \mathrm{e}$ & $17.64 \mathrm{e}$ & $18.44 \mathrm{e}$ & $15.17 \mathrm{e}$ & $15.65 \mathrm{e}$ \\
\hline $200+0.5 \%$ & $91.24 b$ & $91.89 \mathrm{c}$ & $67.93 b$ & $69.12 b$ & $19.58 b$ & $20.17 b$ & $17.00 \mathrm{~b}$ & $17.98 \mathrm{~b}$ \\
\hline 300 & $88.08 \mathrm{~d}$ & $93.74 b$ & $66.90 \mathrm{c}$ & $767.90 \mathrm{c}$ & $19.12 \mathrm{c}$ & $19.70 \mathrm{c}$ & $16.54 \mathrm{c}$ & $17.59 \mathrm{c}$ \\
\hline $\begin{array}{c}300+0.5 \% \\
\text { F-test }\end{array}$ & $\begin{array}{c}96.16 \mathrm{~b} \\
* *\end{array}$ & $\begin{array}{l}97.96 \mathrm{a} \\
* *\end{array}$ & $\begin{array}{l}75.30 \mathrm{a} \\
* *\end{array}$ & $\begin{array}{c}75.51 \mathrm{a} \\
* *\end{array}$ & $\begin{array}{l}21.00 \mathrm{a} \\
* *\end{array}$ & $\begin{array}{l}21.69 \mathrm{a} \\
* *\end{array}$ & $\begin{array}{l}20.29 \mathrm{a} \\
\quad * *\end{array}$ & $\begin{array}{l}21.18 \mathrm{a} \\
* *\end{array}$ \\
\hline
\end{tabular}

Interaction

$(\mathrm{N} \times \mathrm{D})$

$*$ = Significant at 0.05 level, $* *=$ Significant at 0.01 level and NS = Not significant. Means in the same column designated by the same letter are not significantly different at $5 \%$ level

\section{Growth regulators effect}

Otherwise, there were highly significant differences among the doses of $\mathrm{GA}_{3}$ application for plant height $(\mathrm{cm})$, panicle exsertion $(\%)$, panicle length $(\mathrm{cm})$ and number of fertile tillers hill ${ }^{-1}$ in both seasons. The highest values for plant height, panicle exsertion, panicle length and number of fertile tillers hill ${ }^{-1}$ were obtained with the doses of $300 \mathrm{~g} \mathrm{GA}_{3}+0.5 \%$ boric acid ha- in both seasons. The lowest values for plant height, panicle exsertion, panicle length and number of fertile tillers hill ${ }^{-1}$ were found under control treatment in both seasons.

\section{Interaction effect}

The interaction between $\mathrm{N}$ levels and doses of $\mathrm{GA}_{3}$ application was highly significant for plant height $(\mathrm{cm})$, panicle exsertion $(\%)$, panicle length $(\mathrm{cm})$ and number of fertile tillers hill ${ }^{-1}$ in both seasons. The results in Table 4 showed that the 
combination between $\mathrm{N}$ level at the rate of $165 \mathrm{~kg} \mathrm{~N}^{-1}$ with the $300 \mathrm{~g} \mathrm{GA}_{3}+0.5 \%$ B.A ha ${ }^{-1}$ produced significantly highest values of plant height, panicle exsertion, panicle length and number of fertile tillers hill ${ }^{-1}$ in both seasons.

Table 4. Effect of $N$ levels and $G A_{3}+B$. A. foliar application, as well as their interaction on plant characteristics during 2019 and 2020 seasons

\begin{tabular}{|c|c|c|c|c|c|c|c|c|c|}
\hline \multirow{2}{*}{$\begin{array}{c}\text { N levels } \\
\left(\mathrm{kg} \mathrm{N} \mathrm{ha}^{-1}\right)\end{array}$} & \multirow{2}{*}{$\begin{array}{c}\text { GA }+ \text { B.A } \\
\left(\mathrm{g} \mathrm{ha}^{-1}\right) \\
\text { dosage }(\mathrm{D})^{2}\end{array}$} & \multicolumn{2}{|c|}{$\begin{array}{l}\text { Plant height } \\
\text { (cm) }\end{array}$} & \multicolumn{2}{|c|}{$\begin{array}{c}\text { Panicle exsertion } \\
(\%)\end{array}$} & \multicolumn{2}{|c|}{$\begin{array}{c}\text { Panicle length } \\
(\mathrm{cm})\end{array}$} & \multicolumn{2}{|c|}{$\begin{array}{l}\text { Number of fertile } \\
\text { tillers hill }^{-1}\end{array}$} \\
\hline & & 2019 & 2020 & 2019 & 2020 & 2019 & 2020 & 2019 & 2020 \\
\hline \multirow{7}{*}{0} & 0 & $74.79 x$ & $77.59 \mathrm{~s}$ & $32.29 \mathrm{~s}$ & $33.35 \mathrm{t}$ & $15.35 p$ & $15.35 p$ & $10.12 q$ & $11.17 \mathrm{r}$ \\
\hline & 150 & $80.38 \mathrm{v}$ & $82.41 \mathrm{q}$ & 56.360 & $57.14 p$ & $16.24 \mathrm{o}$ & $16.24 \mathrm{o}$ & $11.59 \mathrm{p}$ & $12.72 \mathrm{o}$ \\
\hline & $150+0.5 \%$ & $84.66 r$ & 86.18klm & $64.17 \mathrm{j}$ & $63.35 \mathrm{k}$ & $16.62 n$ & $16.62 n$ & $12.62 n$ & $14.02 n$ \\
\hline & 200 & $84.09 \mathrm{~s}$ & $85.75 \mathrm{mn}$ & $62.38 \mathrm{k}$ & $62.02 \mathrm{k}$ & $16.14 \mathrm{o}$ & $16.14 \mathrm{o}$ & $12.15 \mathrm{o}$ & $13.97 n$ \\
\hline & $200+0.5 \%$ & $86.65 n$ & $88.38 \mathrm{j}$ & $68.18 \mathrm{f}$ & $67.20 \mathrm{~g}$ & $17.12 \mathrm{~lm}$ & $17.121 \mathrm{~m}$ & 13.951 & $15.17 \mathrm{k}$ \\
\hline & 300 & $85.97 \mathrm{o}$ & $88.02 \mathrm{j}$ & $67.43 \mathrm{~g}$ & $66.30 \mathrm{~h}$ & $17.01 \mathrm{~m}$ & $17.01 \mathrm{~m}$ & $13.27 \mathrm{~m}$ & $15.02 \mathrm{kl}$ \\
\hline & $300+0.5 \%$ & $88.01 \mathrm{j}$ & $89.98 \mathrm{~h}$ & $69.16 \mathrm{e}$ & $68.42 \mathrm{f}$ & $18.29 \mathrm{~h}$ & $18.29 \mathrm{~h}$ & $14.84 \mathrm{jk}$ & $16.03 \mathrm{j}$ \\
\hline \multirow{7}{*}{55} & 0 & $78.05 \mathrm{w}$ & $80.20 \mathrm{r}$ & $34.02 \mathrm{r}$ & $34.41 \mathrm{~s}$ & $16.20 \mathrm{o}$ & $16.20 \mathrm{o}$ & $11.72 p$ & $12.31 \mathrm{q}$ \\
\hline & 150 & $83.64 t$ & 83.930 & $57.38 n$ & 58.310 & $17.36 \mathrm{kl}$ & $17.36 \mathrm{kl}$ & $13.25 \mathrm{~m}$ & $13.28 \mathrm{o}$ \\
\hline & $150+0.5 \%$ & $85.45 p$ & $86.35 \mathrm{kl}$ & $65.61 \mathrm{i}$ & $64.48 j$ & $17.64 \mathrm{jk}$ & $17.64 \mathrm{jk}$ & $14.96 \mathrm{ij}$ & $14.811 \mathrm{~lm}$ \\
\hline & 200 & $85.07 \mathrm{q}$ & $86.031 \mathrm{mn}$ & $64.01 \mathrm{j}$ & $63.36 \mathrm{k}$ & $17.41 \mathrm{kl}$ & $17.41 \mathrm{k}$ & $14.69 \mathrm{jk}$ & $14.16 n$ \\
\hline & $200+0.5 \%$ & $87.60 \mathrm{k}$ & 88.46j & $65.48 \mathrm{i}$ & $68.20 \mathrm{f}$ & $18.78 \mathrm{~g}$ & $18.78 \mathrm{~g}$ & $15.76 \mathrm{~h}$ & $16.86 \mathrm{i}$ \\
\hline & 300 & $87.01 \mathrm{~m}$ & $88.11 \mathrm{j}$ & $64.47 j$ & $67.4 \mathrm{~g}$ & $18.42 \mathrm{~h}$ & $18.42 \mathrm{~h}$ & $15.27 \mathrm{i}$ & $16.16 \mathrm{j}$ \\
\hline & $300+0.5 \%$ & $90.22 \mathrm{i}$ & $92.39 \mathrm{~g}$ & $75.3 c$ & $76.83 \mathrm{c}$ & $19.48 \mathrm{e}$ & $19.48 \mathrm{e}$ & $17.53 \mathrm{ef}$ & $18.21 \mathrm{f}$ \\
\hline \multirow{7}{*}{110} & 0 & $80.84 u$ & $83.47 p$ & $34.64 q$ & $35.28 \mathrm{r}$ & $17.081 \mathrm{~m}$ & $17.081 \mathrm{~m}$ & $12.74 n$ & 13.310 \\
\hline & 150 & $85.65 p$ & $86.59 \mathrm{k}$ & $58.38 \mathrm{~m}$ & $59.28 n$ & $17.88 \mathrm{ij}$ & $17.88 \mathrm{ij}$ & $14.64 \mathrm{jk}$ & $14.67 \mathrm{~m}$ \\
\hline & $150+0.5 \%$ & $88.01 \mathrm{j}$ & $89.94 \mathrm{~h}$ & $67.21 \mathrm{~g}$ & $66.10 \mathrm{~h}$ & $18.33 \mathrm{~h}$ & $18.33 \mathrm{~h}$ & $16.33 \mathrm{~g}$ & $17.35 \mathrm{~h}$ \\
\hline & 200 & 87.351 & $89.04 i$ & $66.37 \mathrm{~h}$ & $65.38 \mathrm{i}$ & $18.01 \mathrm{i}$ & $18.01 \mathrm{i}$ & $16.03 \mathrm{gh}$ & $17.23 \mathrm{~h}$ \\
\hline & $200+0.5 \%$ & $92.37 \mathrm{~g}$ & $94.28 \mathrm{e}$ & $68.28 \mathrm{f}$ & $69.47 \mathrm{e}$ & $20.23 d$ & $20.23 d$ & $17.66 \mathrm{ef}$ & $18.56 \mathrm{e}$ \\
\hline & 300 & $91.10 \mathrm{~h}$ & $93.1 \mathrm{f}$ & $67.48 \mathrm{~g}$ & $68.41 \mathrm{f}$ & $19.0 \mathrm{fg}$ & $19.00 \mathrm{fg}$ & $17.29 \mathrm{f}$ & $18.17 \mathrm{f}$ \\
\hline & $300+0.5 \%$ & $96.27 \mathrm{~d}$ & $97.29 \mathrm{c}$ & $76.41 \mathrm{~b}$ & $77.30 \mathrm{~b}$ & $22.63 b$ & $22.63 b$ & $23.51 \mathrm{~b}$ & $24.28 \mathrm{~b}$ \\
\hline \multirow{7}{*}{165} & 0 & $86.84 \mathrm{mn}$ & $85.58 \mathrm{n}$ & $35.46 \mathrm{p}$ & $36.20 q$ & $17.75 \mathrm{ij}$ & $17.75 \mathrm{ij}$ & $14.42 \mathrm{k}$ & $14.56 \mathrm{~m}$ \\
\hline & 150 & $90.28 \mathrm{i}$ & $88.49 \mathrm{j}$ & 59.451 & $60.39 \mathrm{~m}$ & $18.40 \mathrm{~h}$ & $18.40 \mathrm{~h}$ & $15.32 \mathrm{i}$ & $16.33 \mathrm{j}$ \\
\hline & $150+0.5 \%$ & $94.19 \mathrm{e}$ & $93.28 \mathrm{f}$ & $68.59 f$ & $67.48 \mathrm{~g}$ & $19.14 \mathrm{f}$ & $19.14 \mathrm{f}$ & $18.13 d$ & $17.81 \mathrm{~g}$ \\
\hline & 200 & $93.38 \mathrm{f}$ & $92.66 \mathrm{~g}$ & $67.42 \mathrm{~g}$ & $66.42 \mathrm{~h}$ & $19.01 \mathrm{fg}$ & $19.01 \mathrm{fg}$ & $17.80 \mathrm{e}$ & $17.23 \mathrm{~h}$ \\
\hline & $200+0.5 \%$ & $98.33 b$ & $96.44 d$ & $69.78 \mathrm{~d}$ & $71.60 \mathrm{~d}$ & $22.20 \mathrm{c}$ & $22.20 \mathrm{c}$ & $20.65 c$ & $21.32 \mathrm{c}$ \\
\hline & 300 & $97.16 \mathrm{c}$ & $105.75 b$ & $68.24 \mathrm{f}$ & $69.51 \mathrm{e}$ & $22.08 \mathrm{c}$ & $22.08 \mathrm{c}$ & $20.35 c$ & $21.03 \mathrm{~d}$ \\
\hline & $300+0.5 \%$ & $110.16 \mathrm{a}$ & $112.19 \mathrm{a}$ & $80.33 a$ & $79.51 \mathrm{a}$ & $23.59 \mathrm{a}$ & $23.59 \mathrm{a}$ & $25.27 \mathrm{a}$ & $26.22 \mathrm{a}$ \\
\hline
\end{tabular}

$*=$ Significant at 0.05 level, $* *=$ Significant at 0.01 level and NS $=$ Not significant. Means in the same column designated by the same letter are not significantly different at $5 \%$ level

\section{Nitrogen levels effect}

The effect of $\mathrm{N}$ levels, doses of $\mathrm{GA}_{3}$ application and their interaction on panicle weight (g), seed set (\%), harvest index (\%) and seed yield $\left(\mathrm{t} \mathrm{ha}^{-1}\right)$ are given in Table 5. The highest values for panicle weight, seed set, harvest index and seed yield were obtained with application of $165 \mathrm{~kg} \mathrm{~N} \mathrm{ha}^{-1}$ in both season, while, the lowest values for 
panicle weight, seed set, harvest index and seed yield were found under control treatment.

\section{Growth regulators effect}

There were highly significant differences among the doses of $\mathrm{GA}_{3}$ application for panicle weight $(\mathrm{g})$, seed set $(\%)$, harvest index $(\%)$ and seed yield $\left(\mathrm{t} \mathrm{ha}^{-1}\right)$ in both seasons. The highest values for panicle weight, seed set, harvest index and seed yield were obtained with the application of $300 \mathrm{~g} \mathrm{GA}_{3}+0.5 \% \mathrm{~B}$. $\mathrm{A} \mathrm{ha}^{-1}$ in both seasons. The lowest values for panicle weight, seed set, harvest index and seed yield were found under control treatment in both seasons.

Table 5. Effect of $\mathrm{N}$ levels and $\mathrm{GA}_{3}+$ B.A. dosage application and their interactions on panicle weight, seed set, harvest index and seed yield during 2019 and 2020 seasons

\begin{tabular}{|c|c|c|c|c|c|c|c|c|}
\hline \multirow{2}{*}{$\begin{array}{l}\text { Main effect and } \\
\text { interaction }\end{array}$} & \multicolumn{2}{|c|}{$\begin{array}{c}\text { Panicle weight } \\
\text { (g) }\end{array}$} & \multicolumn{2}{|c|}{$\begin{array}{l}\text { Seed set } \\
(\%)\end{array}$} & \multicolumn{2}{|c|}{$\begin{array}{c}\text { Harvest index } \\
(\%)\end{array}$} & \multicolumn{2}{|c|}{$\begin{array}{c}\text { Seed yield } \\
\left(t \text { ha }^{-1}\right)\end{array}$} \\
\hline & 2019 & 2020 & 2019 & 2020 & 2019 & 2020 & 2019 & 2020 \\
\hline \multicolumn{9}{|l|}{ N levels (kg N ha-1) } \\
\hline 0 & $1.61 \mathrm{~d}$ & $1.67 \mathrm{~d}$ & $21.62 d$ & $23.06 \mathrm{~d}$ & $12.96 \mathrm{~d}$ & $14.03 \mathrm{~d}$ & $0.753 d$ & $0.849 \mathrm{~d}$ \\
\hline 55 & $1.99 \mathrm{c}$ & $2.01 \mathrm{c}$ & $23.25 c$ & $24.86 \mathrm{c}$ & $14.60 \mathrm{c}$ & $15.72 \mathrm{c}$ & $0.859 \mathrm{c}$ & $1.057 \mathrm{c}$ \\
\hline 110 & $2.28 \mathrm{~b}$ & $2.43 b$ & $25.44 b$ & $26.87 b$ & $15.74 b$ & $16.95 b$ & $1.155 b$ & $1.302 \mathrm{~b}$ \\
\hline 165 & $2.49 \mathrm{a}$ & $2.69 \mathrm{a}$ & $27.94 \mathrm{a}$ & $28.92 \mathrm{a}$ & $17.57 \mathrm{a}$ & $18.77 \mathrm{a}$ & $1.496 \mathrm{a}$ & 1.588 \\
\hline F-test & $* *$ & $* *$ & $* *$ & $* *$ & $* *$ & $* *$ & $* *$ & $* *$ \\
\hline \multicolumn{9}{|c|}{$\mathrm{GA}_{3}+$ B.A $\left(\mathrm{g} \mathrm{ha}^{-1}\right)$ dosage (D) } \\
\hline 0 & $1.62 \mathrm{f}$ & $1.65 \mathrm{~g}$ & $17.75 \mathrm{~g}$ & $18.92 \mathrm{e}$ & $11.36 \mathrm{~g}$ & $12.77 \mathrm{~g}$ & $0.735 \mathrm{~g}$ & $0.835 f$ \\
\hline 150 & $1.82 \mathrm{e}$ & $1.86 \mathrm{f}$ & $19.89 f$ & $21.18 \mathrm{~d}$ & $13.07 \mathrm{f}$ & $14.07 f$ & $0.850 \mathrm{f}$ & $0.975 \mathrm{e}$ \\
\hline $150+0.5 \%$ & $1.95 \mathrm{~d}$ & $2.07 \mathrm{~d}$ & $23.54 \mathrm{~d}$ & $24.49 \mathrm{c}$ & $14.84 d$ & $16.19 \mathrm{~d}$ & $1.015 \mathrm{~d}$ & $1.083 \mathrm{~d}$ \\
\hline 200 & $1.85 \mathrm{e}$ & $1.97 \mathrm{e}$ & $23.13 \mathrm{e}$ & $24.30 \mathrm{c}$ & $14.49 \mathrm{e}$ & $15.84 \mathrm{e}$ & $0.990 \mathrm{e}$ & $1.065 \mathrm{~d}$ \\
\hline $200+0.5 \%$ & $2.35 \mathrm{~b}$ & $2.44 \mathrm{~b}$ & $27.91 b$ & $29.19 b$ & $16.93 b$ & $18.03 b$ & $1.231 \mathrm{~b}$ & $1.430 \mathrm{~b}$ \\
\hline 300 & $2.24 \mathrm{c}$ & $2.38 \mathrm{c}$ & $27.49 \mathrm{c}$ & $29.54 b$ & $16.60 \mathrm{c}$ & $17.61 \mathrm{c}$ & $1.209 \mathrm{c}$ & $1.335 \mathrm{c}$ \\
\hline $\begin{array}{c}300+0.5 \% \\
\text { F-test }\end{array}$ & $\begin{array}{l}2.84 \mathrm{a} \\
* *\end{array}$ & $\begin{array}{c}3.03 \mathrm{a} \\
* *\end{array}$ & $\begin{array}{c}32.23 \mathrm{a} \\
* *\end{array}$ & $\begin{array}{c}33.67 \mathrm{a} \\
* *\end{array}$ & $\begin{array}{c}19.26 \mathrm{a} \\
* *\end{array}$ & $\begin{array}{c}20.33 \mathrm{a} \\
* *\end{array}$ & $\begin{array}{c}1.433 \mathrm{a} \\
* *\end{array}$ & $\begin{array}{c}1.670 \mathrm{a} \\
* *\end{array}$ \\
\hline
\end{tabular}

\section{Interaction}

$(\mathrm{N} \times \mathrm{D})$

$*=$ Significant at 0.05 level, $* *=$ Significant at 0.01 level and NS = Not significant. Means in the same column designated by the same letter are not significantly different at $5 \%$ level

\section{Interaction effect}

The interaction between $\mathrm{N}$ levels and doses of $\mathrm{GA}_{3}$ application was highly significant for panicle weight, seed set, harvest index and seed yield in both seasons. The results in Table 6 showed that the combination of $165 \mathrm{~kg} \mathrm{~N} \mathrm{ha}^{-1}$ with the doses $300 \mathrm{~g} \mathrm{GA}_{3}+0.5 \%$ B.A ha ${ }^{-1}$ produced significantly highest values of panicle weight, seed set, harvest index and seed yield in both seasons. While the lowest values were recorded in control treatment in both seasons. 
Table 6. Effect of $N$ levels and $G A_{3}+$ B.A. foliar application, as well as their interaction on panicle characteristics and yield during 2019 and 2020 seasons

\begin{tabular}{|c|c|c|c|c|c|c|c|c|c|}
\hline \multirow{2}{*}{$\begin{array}{r}\text { N levels } \\
\left(\mathrm{kg} \mathrm{N} \mathrm{ha}^{-1}\right.\end{array}$} & \multirow{2}{*}{$\begin{array}{c}\mathrm{GA}_{3}+\mathrm{B} . \mathrm{A} \\
\left(\mathrm{g} \mathrm{ha}^{-1}\right) \\
\text { dosage }^{(D)}\end{array}$} & \multicolumn{2}{|c|}{$\begin{array}{c}\text { Panicle weight } \\
\text { (g) }\end{array}$} & \multicolumn{2}{|c|}{$\begin{array}{c}\text { Seed set } \\
(\%)\end{array}$} & \multicolumn{2}{|c|}{$\begin{array}{c}\text { Harvest index } \\
(\%)\end{array}$} & \multicolumn{2}{|c|}{$\begin{array}{c}\text { Seed yield } \\
\left(\mathbf{t ~ h a}^{-1}\right)\end{array}$} \\
\hline & & 2019 & 2020 & 2019 & \begin{tabular}{|l|}
2020 \\
\end{tabular} & 2019 & 2020 & 2019 & 2020 \\
\hline \multirow{7}{*}{0} & 0 & $1.20 \mathrm{p}$ & $1.38 \mathrm{o}$ & $16.22 q$ & 17.230 & $10.55 n$ & $11.22 \mathrm{~m}$ & $0.530 \mathrm{n}$ & 0.6701 \\
\hline & 150 & $1.24 p$ & $1.29 p$ & $18.13 \mathrm{o}$ & $18.48 \mathrm{mno}$ & $11.25 \mathrm{~m}$ & 12.381 & $0.620 \mathrm{~m}$ & $0.720 \mathrm{kl}$ \\
\hline & $150+0.5 \%$ & $1.43 \mathrm{o}$ & $1.47 \mathrm{n}$ & $20.11 \mathrm{~m}$ & $22.05 \mathrm{klm}$ & $12.23 \mathrm{k}$ & $13.48 \mathrm{k}$ & 0.7161 & $0.790 \mathrm{i}-1$ \\
\hline & 200 & $1.31 \mathrm{p}$ & $1.39 \mathrm{o}$ & $19.77 \mathrm{~m}$ & $21.87 \mathrm{klm}$ & 11.791 & $13.02 \mathrm{k}$ & 0.7001 & $0.776 \mathrm{i}-1$ \\
\hline & $200+0.5 \%$ & $1.98 \mathrm{il}$ & $1.94 \mathrm{k}$ & $24.67 \mathrm{i}$ & 26.33f-i & $14.40 \mathrm{i}$ & $15.73 \mathrm{~h}$ & $0.880 \mathrm{hi}$ & $0.950 \mathrm{hi}$ \\
\hline & 300 & $1.92 \mathrm{jkl}$ & $1.90 \mathrm{k}$ & $23.81 \mathrm{k}$ & $25.83 \mathrm{~g}-\mathrm{j}$ & $14.07 \mathrm{i}$ & $15.14 \mathrm{i}$ & $0.850 \mathrm{j}$ & 0.920hij \\
\hline & $300+0.5 \%$ & $2.19 \mathrm{fg}$ & $2.31 \mathrm{~h}$ & $28.64 \mathrm{e}$ & $29.63 \mathrm{cde}$ & $16.42 \mathrm{~g}$ & $17.24 \mathrm{~g}$ & $0.980 \mathrm{~g}$ & $1.116 \mathrm{fg}$ \\
\hline \multirow{7}{*}{55} & 0 & $1.63 n$ & $1.56 \mathrm{~m}$ & $17.35 p$ & $18.44 \mathrm{no}$ & $10.79 \mathrm{~m}$ & 12.321 & $0.610 \mathrm{~m}$ & $0.750 \mathrm{jkl}$ \\
\hline & 150 & $1.831 \mathrm{~m}$ & 1.701 & $19.16 n$ & $20.201 \mathrm{mn}$ & $12.51 \mathrm{k}$ & $13.43 \mathrm{k}$ & 0.7101 & $0.850 \mathrm{ijk}$ \\
\hline & $150+0.5 \%$ & $1.93 \mathrm{jkl}$ & $1.95 \mathrm{k}$ & 22.541 & 23.88ijk & $14.35 \mathrm{i}$ & $15.70 \mathrm{~h}$ & $0.843 \mathrm{j}$ & 0.920hij \\
\hline & 200 & $1.87 \mathrm{kl}$ & $1.90 \mathrm{k}$ & 22.181 & $23.18 \mathrm{jk}$ & $14.03 \mathrm{i}$ & $15.11 \mathrm{i}$ & $0.810 \mathrm{k}$ & $0.903 \mathrm{hij}$ \\
\hline & $200+0.5 \%$ & $2.12 \mathrm{f}-\mathrm{i}$ & $2.18 \mathrm{ij}$ & $26.18 \mathrm{~h}$ & $28.79 \mathrm{c}-\mathrm{f}$ & $16.21 \mathrm{~g}$ & $17.07 \mathrm{~g}$ & $0.980 \mathrm{~g}$ & $1.410 \mathrm{~cd}$ \\
\hline & 300 & $2.01 \mathrm{~h}-\mathrm{k}$ & $2.12 \mathrm{j}$ & $26.02 \mathrm{~h}$ & $28.20 \mathrm{~d}-\mathrm{g}$ & $16.09 \mathrm{~g}$ & $16.99 \mathrm{~g}$ & $0.946 \mathrm{gh}$ & $1.133 \mathrm{fg}$ \\
\hline & $300+0.5 \%$ & $2.01 \mathrm{~d}$ & $2.67 \mathrm{e}$ & $29.29 \mathrm{~d}$ & $31.29 \mathrm{c}$ & $18.25 \mathrm{~d}$ & $19.42 \mathrm{~d}$ & $1.116 \mathrm{f}$ & $1.433 \mathrm{~cd}$ \\
\hline \multirow{7}{*}{110} & 0 & $1.73 \mathrm{mn}$ & 1.681 & $18.13 n$ & $19.49 \mathrm{mno}$ & $11.36 \mathrm{~m}$ & $13.34 \mathrm{k}$ & $0.853 \mathrm{j}$ & $0.860 \mathrm{ijk}$ \\
\hline & 150 & $2.01 \mathrm{~h}-\mathrm{k}$ & $2.15 \mathrm{j}$ & $20.09 \mathrm{~m}$ & $22.50 \mathrm{kl}$ & $13.38 \mathrm{j}$ & $14.27 \mathrm{j}$ & $0.910 \mathrm{hi}$ & $1.033 \mathrm{gh}$ \\
\hline & $150+0.5 \%$ & $2.16 \mathrm{fgh}$ & $2.38 \mathrm{~h}$ & $24.65 \mathrm{i}$ & 25.40hij & $15.41 \mathrm{~h}$ & $16.90 \mathrm{~g}$ & $0.983 \mathrm{~g}$ & $1.213 \mathrm{ef}$ \\
\hline & 200 & $2.07 \mathrm{~g}-\mathrm{j}$ & $2.24 \mathrm{i}$ & $24.18 \mathrm{j}$ & 25.09hij & $15.10 \mathrm{~h}$ & $16.14 \mathrm{~h}$ & $0.95 \mathrm{gh}$ & $1.190 \mathrm{efg}$ \\
\hline & $200+0.5 \%$ & $2.54 \mathrm{~d}$ & $2.66 \mathrm{e}$ & $28.40 \mathrm{e}$ & $30.20 \mathrm{~cd}$ & $17.61 \mathrm{e}$ & $18.84 \mathrm{e}$ & $1.363 \mathrm{~d}$ & $1.516 \mathrm{c}$ \\
\hline & 300 & $2.40 \mathrm{e}$ & $2.58 \mathrm{f}$ & $28.01 \mathrm{f}$ & $30.09 \mathrm{~cd}$ & $17.14 \mathrm{ef}$ & $17.97 \mathrm{f}$ & $1.340 \mathrm{~d}$ & $1.476 \mathrm{c}$ \\
\hline & $300+0.5 \%$ & $3.04 \mathrm{~b}$ & $3.28 \mathrm{~b}$ & $34.60 \mathrm{~b}$ & $35.32 \mathrm{~b}$ & $20.21 \mathrm{~b}$ & $21.20 \mathrm{~b}$ & $1.690 \mathrm{~b}$ & $1.830 \mathrm{~b}$ \\
\hline \multirow{7}{*}{165} & 0 & $1.89 \mathrm{kl}$ & 1.96 & $19.29 n$ & $20.501 \mathrm{mn}$ & $12.73 \mathrm{k}$ & $14.21 \mathrm{j}$ & $0.946 \mathrm{gh}$ & $1.063 \mathrm{fgh}$ \\
\hline & 150 & $2.17 \mathrm{fgh}$ & $2.32 \mathrm{~h}$ & 22.201 & & $15.15 \mathrm{~h}$ & $16.20 \mathrm{~h}$ & $1.160 \mathrm{e}$ & $1.296 \mathrm{de}$ \\
\hline & $150+0.5 \%$ & $2.26 f$ & $2.38 \mathrm{~h}$ & $26.86 \mathrm{~g}$ & $27.43 e-h$ & $17.38 \mathrm{ef}$ & $18.68 \mathrm{e}$ & $1.516 \mathrm{c}$ & $1.410 \mathrm{~cd}$ \\
\hline & 200 & $2.13 \mathrm{f}-\mathrm{i}$ & $2.36 \mathrm{~h}$ & $26.36 \mathrm{~h}$ & $27.06 \mathrm{e}-\mathrm{h}$ & $17.02 \mathrm{f}$ & $18.05 \mathrm{f}$ & $1.500 \mathrm{c}$ & $1.390 \mathrm{~cd}$ \\
\hline & $200+0.5 \%$ & $2.74 \mathrm{c}$ & $2.97 \mathrm{c}$ & $32.38 \mathrm{c}$ & $31.45 \mathrm{c}$ & $19.50 \mathrm{c}$ & $20.47 \mathrm{c}$ & $1.703 b$ & $1.846 \mathrm{~b}$ \\
\hline & 300 & $2.68 \mathrm{c}$ & $2.87 \mathrm{~d}$ & $32.12 \mathrm{c}$ & $34.02 \mathrm{~b}$ & $19.11 \mathrm{c}$ & $20.34 \mathrm{c}$ & $1.700 \mathrm{~b}$ & $1.810 \mathrm{~b}$ \\
\hline & $300+0.5 \%$ & $3.59 \mathrm{a}$ & $3.85 \mathrm{a}$ & $36.41 \mathrm{a}$ & $38.43 \mathrm{a}$ & $22.13 \mathrm{a}$ & $23.49 \mathrm{a}$ & $1.946 \mathrm{a}$ & $2.303 \mathrm{a}$ \\
\hline
\end{tabular}

$*=$ Significant at 0.05 level, $* *=$ Significant at 0.01 level and NS $=$ Not significant. Means in the same column designated by the same letter are not significantly different at $5 \%$ level

\section{Discussion}

The improved in rice yield attributes with increasing $\mathrm{N}$ levels mostly due to the function of $\mathrm{N}$ on improving rice growth, photosynthesis, internodes elongation led to extra panicle formation during the productive stage, produce maximum spikelets per panicle as well as grain filling accordingly, the weight of grain was high. The rice grain yield enhancement could be due to the role of $\mathrm{N}$ in increasing grain yield attributes i.e., panicles hill ${ }^{-1}$, panicle length, number of filled grains per panicle and panicle weight (Sorour et al., 2016; Gewaily et al., 2018). Haque et al. (2006) reported that irrespective of rice cultivars, the days requested to heading and maturity significantly increased with the increasing the level of $\mathrm{N}$ application. Accelerated vegetative growth could be a reason for delaying heading and crop maturity with the increasing the of $\mathrm{N}$ fertilizer rates. Also, Elankavi et al., 2009) reported that the delayed heading with higher $\mathrm{N}$ rates may be due to more vegetative growth, which resulted in, delayed maturity. The results are in 
agreements with those obtained by Gharib et al. (2011) and Fu and Yang (2011). Cui et al. (2010) reported that, the suitable $\mathrm{N}$ application rate of Japonica super rice in the northern China was $147 \mathrm{~kg} \mathrm{ha}^{-1}$, while, Li et al. (2011) found that, $\mathrm{N}$ application rate was $270 \mathrm{~kg} \mathrm{ha}^{-1}$ in order to obtain the yield of $7.5 \mathrm{t} \mathrm{ha}^{-1}$ studied the super rice Xinliangyou 6 in the southern China and found a suitable. Zhu et al. (2015) investigated the N application rate on grain yield and quality of Japonic $a$ rice Wuyunjing27 and found that, the optimum amount of $\mathrm{N}$ fertilizer was $225-300 \mathrm{~kg} \mathrm{ha}^{-1}$. The variations in the suitable $\mathrm{N}$ application rates in the above studies were due to differences in rice varieties and ecological zones.

Poor panicle exsertion of male sterile lines is a major problem in hybrid rice seed production. Hence, the foliar application of $\mathrm{GA}_{3}$ at the start of panicle emergence has been widely adopted as an essential technology for improving plant height and panicle exsertion. Godha et al. (2020) reported that applied auxins (i.e., NAA, GA 3 and ascorbic acid) increased the highest value of panicle exsertion and the increases ranged between $87.80 \%$ and $88.67 \%$ when rice plants of A-line were treated with $200 \mathrm{~g} \mathrm{GA}_{3} \mathrm{ha}^{-1}$ of. The results indicated that, the addition of the $0.5 \%$ boric acid with $300 \mathrm{~g} \mathrm{GA}_{3} \mathrm{ha}^{-1}$, positively improved the morphological rice traits. GabAllah (2004) reported that, the highest value of leaf angle was ranged between 41.67 to 44.01 when rice plants of A line were treated with $200 \mathrm{~g} \mathrm{GA}_{3} \mathrm{ha}^{-1}$. The results are in agreement with those reported by El-Mowafi et al. (2016). The application of $\mathrm{GA}_{3}$ influences panicle exsertion, spikelet opening angle and other floral traits which increases outcrossing rate of CMS lines leading higher yield. Rahman et al. (2010) reported that, application of $\mathrm{GA}_{3}$ at the rate of $150 \mathrm{~g} \mathrm{ha}^{-1}$ increased panicle exsertion and out crossing rate and seed yield compared with without $\mathrm{GA}_{3}$. Zhen et al. (2018) found that $\mathrm{GA}_{3}$ application at the time flowering produced hybrid seeds with a higher seedling vigor index. Additionally, it has been documented that $\mathrm{GA}_{3}$ stimulates the synthesis and production of hydrolases, resulting in the germination of seeds. Therefore, $\mathrm{GA}_{3}$ application may affect on seed vigor during hybrid rice seed production. During hybrid rice seed production, the foliar application of $\mathrm{GA}_{3}$ at the start of panicle emergence improving panicle exsertion of male sterile lines. Moreover, many investigations were conducted in southern China reported that, spraying of $\mathrm{GA}_{3}$ at $15-30 \mathrm{~g}$ for several times after anthesis can act stigma properties, including stigma vigor which ultimately influence the out-crossing rate and seed yield potential. Previous studies have reported the erect of $\mathrm{GA}_{3}$ application on seed yield during hybrid rice seed production. However, knowledge is limited about the effect of $\mathrm{GA}_{3}$ application after anthesis on the seed vigor of hybrid rice. We hypothesized that a low concentration of $\mathrm{GA}_{3}$ application after anthesis can enhance stigma vigor and correspondingly improve seed vigor in hybrid rice seed production. The filament length of the anther determines the degree of versatility of the anthers i.e., higher the filament length greater the degree of versatility of the anther and higher frequency of pollen dispersal and migration from the male parent to female parent. Xiaomin et al. (2019) observed that $\mathrm{GA}_{3}$ application after anthesis could prolong the duration of seed filling and enhance the sink capacity, correspondingly improving grain weight and out-crossing, and eventually increasing the seed yield. Consequently, the higher seed yield under the $\mathrm{GA}_{3}$ application with $30 \mathrm{~g} \mathrm{ha}^{-1}$ was mainly ascribed to the significantly higher 1000 -grain weight and out-crossing rate. The increase in yield with the application of $\mathrm{GA}_{3}$ might be due to increased yield attributes, which in turn resulted from effective translocation of photosynthates. The plant growth hormones also increase mobilization of reserve food materials to the developing sink through increase in hydrolyzing and oxidizing enzyme activities and leads to yield increases (Jayachandran et al., 2000; Muniandi et al., 2018). 


\section{Conclusions}

The article aimed to investigate the effects of various $\mathrm{N}$ doses and foliar $\mathrm{GA}_{3}$ application on the seed yield of hybrid and parent rice grown in Egypt, using a split-plot design with three replications. The results indicated that, $\mathrm{GA}_{3}$ application significantly enhanced the stigma vigor index of the female parent and correspondingly increased the out-crossing rate. Moreover, $\mathrm{GA}_{3}$ application should be applied at a rate of $300 \mathrm{~g} \mathrm{GA}_{3} \mathrm{ha}^{-}$ ${ }^{1}$ to improve seed vigor and increased hybrid rice seed production. Nitrogen application at the level of $165 \mathrm{~kg} \mathrm{~N} \mathrm{ha}^{-1}$ produced the highest seed yield in both seasons. The treatment combination of ( $165 \mathrm{~kg} \mathrm{~N} \mathrm{ha}^{-1}$ and $300 \mathrm{~g} \mathrm{GA}_{3} \mathrm{ha}^{-1}+0.5 \%$ B.A) recorded the best effects for most of the rice characteristics and recommended for achieving higher hybrid rice seed production in Egypt.

\section{REFERENCES}

[1] Bastawisi, A. O., El-Mowafi, H. F., Abo Youssef, M. I., Draz, A. E., Aidy, I. R. M., Maximos, A., Badawi, A. B. (2002): Advances in hybrid rice research and development in the tropics. - Proceedings of the $4^{\text {th }}$ International Symposium on Hybrid Rice, Hanoi, Vietnam, 14-17 May, pp. 257-264.

[2] Cui, Y. F., Lu, T. G., Sun, G. C., Wang, J. R., Li, D. W., Li, D. Q., Li, D. P. (2010): Effects of different nitrogen application levels on the biological yield and $\mathrm{N}$ use efficiency of super rice in northern China. - Journal of Shenyang Agricultural University 41: 550-554.

[3] Elankavi, S., Kuppuswamy, G., Vaiyapuri, V., Raman, R. (2009): Effect of phytohormones on growth and yield of rice. - Oryza 46: 310-313.

[4] El-Mowafi, H. F., El Gammaal, A. A., Arafat, E. F., Abd Elrahman, W. A. (2016): Studies on hybrid rice seed production for Egyptian cytoplasmic genetic male sterile line Sakha 1A/B multiplication. - Journal of Agricultural Research, Kafr El-Sheikh University, Plant Production 42(3).

[5] Fageria, N. K., Baligar, V. C. (2003): Methodology for evaluation of lowland rice genotypes for nitrogen use efficiency. - Journal of Plant Nutrition 26: 1315-1333.

[6] Fu, J., Yang, J. C. (2011): Research advances in physiology of super rice under highyielding cultivation. - Chinese Journal of Rice Science 25: 343-348.

[7] Gaballah, M. M. (2004): Studies on hybrid rice seed production. - M.Sc. Thesis, Faculty of Agriculture, Kafr El-Sheikh, Tanta University, Egypt.

[8] Gewaily, E. E., Ghoneim, A. M., Osman, M. A. (2018): Effects of nitrogen levels on growth, yield and nitrogen use efficiency of some newly released Egyptian rice genotypes. - Open Agriculture 3: 310-318.

[9] Gewaily, E. E., Ghoneim, A. M., Khattab, E. A. (2019): Nano-silicon and nitrogen foliar spray affects the growth, yield and nutrients content of rice. - World Journal Agricultural Sciences 15(6): 367-375.

[10] Gharib, H. S., Metwally, T. M., Naeem, S. S., Gewaily, E. E. (2011): Influence of some stimulating compounds and nitrogen fertilizer levels on growth and yield of hybrid rice. Zagazig Journal Agricultural Research 38: 1-21.

[11] Ghoneim, A. M. (2020): Soil nutrients availability, rice productivity and water saving under deficit irrigation conditions. - Journal of Plant Production, Mansoura University 11(1): 7-16.

[12] Godha, U., Kataria, G., Singh, C., Pandya, Y., Meena, B. (2020): Effect of NAA, GA 3 and ascorbic acid on growth and morpho-physiological parameters of wheat. International Journal of Chemical Studies 8(1): 432-435. 
[13] Gomez, K. A., Gomez, A. A. (1984): Statistical Procedures for Agricultural Research. 2nd Ed. - John Wiley and Sons, Inc. New York.

[14] Hamad, H. S. (2018): Impact of male to female ratio, flag leaf clipping and time of $\mathrm{GA}_{3}$ application on hybrid rice productivity. - Egypt Journal Plant Breeding 22(2): 277-290.

[15] Haque, K. M., Khaliq, S. K., Aktar, J., Shamsuddula, A. M. (2006): Yielding ability of aromatic rice under stacking and non-stacking conditions with different nitrogen fertilization. - Insertional Journal Sustainable Crop Production 1(1): 1-5.

[16] Jayachandran, M., Gopal, N. P., Marimuthu, R. (2000): Performance of hybrid rice cultures under different levels of nitrogen in combination with growth regulators. Madras Agriculture Journal 89: 462-465.

[17] Li, J., Xin, Y., Yuan, P. (2009): Hybrid rice in China in pushing the yield frontier. IFPRI Discussion Paper. Hybrid Rice Technology Development: Ensuring China`s Food Security. International Food Policy Research Institute, Washington, DC, pp. 77-82. http://www.ifpri.org/book-5826/millionsfed/cases/hybridrice.

[18] Li, X. X., Qin, K. X., Shen, F. J., Luo, T. P., Chen, G. L., Qin, R. D., Xiang, H. X., Huang, B. (2011): Effect of different rates of nitrogen application on yield of rice variety Xinliangyou 6. - Journal of Southern Agriculture 42: 521-523.

[19] Muniandi, S. K. M., Hossain, M. A., Abdullah, M. P., Shukor, N. A. A. (2018): Gibberellic acid (GA3) affects growth and development of some selected kenaf Hibiscus cannabinus L.) cultivars. - International Crop Production 118: 180-187.

[20] Parihar, A., Mahesuria, A. A., Chaurasia, P., Pathak, A. R. (2012): Effect of GA3 and other chemicals for increased seed yield of CMS lines in rice. - Journal Plant Breeding 3: 952-955.

[21] Rahman, M. H., Ali, M. H., Hasan, M. J., Kulsum, M. U., Khatun, M. M. (2010): Outcrossing rate in row ratio of restorer and CMS lines for hybrid rice seed production. Eco-friendly Agricultural Journal 3(5): 233-236.

[22] RRTC (2018): Annual Proceeding for the $11^{\text {th }}$ National Rice Program Workshop. - Rice Research and Training Center, Sakha, Kafr El-Sheikh, Egypt.

[23] Shaiful, I. M., Hasanuzzamanb, M., Rokonuzzaman, M., Nahard, K. (2009): Effect of split application of nitrogen fertilizer on physiological parameters of rice genotypes. International Journal of Plant Production 3(1): 51-62.

[24] Shi-Hua, Y., Ben-Yi, C., Jian-Li, W., Wei-Feng, S., Shi-Hua, C. (2006): Review and prospects on rice breeding and extension in China. - Rice Sciences 13: 1-8.

[25] Sorour, F. A., Ragab, A. W., Metwally, T. F., Shafik, A. A. (2016): Effect of planting methods and nitrogen fertilizer rates on the productivity of rice (Oryza sativa L.). Journal of Agricultural Research, Kafr El-Sheikh University, Plant Production 42: 207216.

[26] Xiaomin, W., Zheng, H., Qiyuan, T., Wenwei, M., Junjie, M. (2019): Effects of gibberellic acid application after anthesis on seed vigor of indica hybrid rice (Oryza sativa L.). - Agronomy 9: 861. DOI: 10.3390/agronomy9120861.

[27] Xie, L. H., Ye, D. C., Hu, P. S., Chen, S. Q., Tang, J., Luo, S., Jiao, J. A. (2011): Effects of nitrogen fertilizer application rate and management strategy on grain yield and quality of rice variety Yongyou 6. - Plant Nutrition and Fertilizer Science 17: 784-794.

[28] Yuan, L. P. (2007): Proposal of implementing the planting-three-produce four highyielding project on super hybrid rice. - Hybrid Rice 22: 1-1.

[29] Yuan, L. P. (2010): Progress in Breeding Super Hybrid Rice. - In: Xie, F., Hardy, B. (eds.) Accelerating Hybrid Rice Development. IRRI, Los Banos, Philippines, pp: 3-8.

[30] Zheng, H. B., Wang, X. M., Li, Y. X., Huang, J. F., Tang, Q. Y., Tang, J. W. (2018): The effects of gibberellin application examined by carbon isotope technology. - Seed Sciences Technology 46: 533-546.

[31] Zhu, B. H., Xu, X. J., Xu, Y. F., Shi, Q., Zhang, Y., Zhang, W. F. (2015): Effect of nitrogen application rate on grain yield and quality of mechanical transplanting japonica rice Wuyunjing 27. - China Rice 21: 63-66. 